\title{
26 AN INNOVATIVE MAINTENANCE SOLUTION FOR COMPLEX MACHINERY: THE KOBAS PROJECT CASE
}

\author{
Américo Azevedo 1,2, Paula Silva ', César Toscano', Joao Cardoso 1,2 \\ ${ }^{\prime}$ INESC Porto, Rua Dr. Roberto Frias $n^{\circ} 378,4200-465$, Porto, Portugal \\ ${ }^{2}$ Faculdade de Engenharia da Universidade do Porto, \\ Rua Dr. Roberto Frias, 4200-465, Porto, Portugal \\ ala@fe.up.pt,psilva@inescporto.pt, ctoscano@inescporto.pt,jcard@fe.up.pt
}

\begin{abstract}
This paper presents an innovative approach in supporting the operation of complex equipment. The concept was developed in the context of the KoBaS project (Knowledge Based Customized Services for Traditional Manufacturing Sectors Provided by a Network of High Tech SMEs) whose main objectives are the development of new knowledge based tools for an intelligent use and management of more sophisticated manufacturing machines; and the creation of an innovative extended network of high-tech SMEs for use, customize, support and make business out of the new development tools. As a concrete example the paper presents a KoBaS solution based on the machine Maintenance. This solution allows one to know the machine condition, to detect and diagnose machine failures, to manage ordinary and extraordinary maintenance plans and maintenance work orders and to provide training support for maintenance interventions.
\end{abstract}

\section{INTRODUCTION}

The companies that build, sell and install equipment are increasingly facing the need to develop technologically advanced and consequently more complex equipment, in order to satisfy the demands of high customization of the products processed by them, with low costs, low manufacturing time and high quality levels.

The KoBaS Project (Knowledge Based Customized Services for Traditional Manufacturing Sectors Provided by a Network of High Tech SMEs) is an European project under the Sixth Framework Programme that tends towards a substantial improvement in equipment and industrial machinery, through the development of a set of software components, integrated in their own equipment and functionally directed at the creation of equipment operation programmes (numerical control programmes), analysis and simulation of operation and movement, sequencing of generation operations, configuration of parameters of functioning, maintenance, formation and support to the operation. The project aims also the development of a network business model, made of technology-based SMEs. The main objective is to foster the development and customization of specific solutions required by the 
equipment manufacturers according to the requisites demanded by the market they wish to satisfy.

One of the developing components under the KoBaS project is destined to support, in an innovative way, the maintenance activities inherent to the equipment itself. The integration of several black-boxes fully integrated and resulting from the customization of the respective components in the equipment itself, makes it possible to reduce substantially the preparation and operation time and to simultaneously reduce the associated costs.

The manufacturers of equipment goods with the developing technology under $\mathrm{KoBaS}$ project, can, therefore, offer more complex products regarding their functionalities, but at the same time easier to operate, which noticeably increases the added value to the client.

The aim of this paper is to address the design of an advanced software solution for maintenance management in the scope of a large on-going European Project (KoBaS Consortium, 2006).

The reminder of this paper is organized as follows. After this introduction, next section presents the project in which the system here described is being developed. The third section presents in particular one of the components in development: the maintenance component. The fourth section addresses the technology and development framework selected for the implementation phase. The fifth and last section concludes the paper.

\section{OVERVIEW OF KoBaS PROJECT APPROACH}

The concepts and component architecture presented in this paper have been conceived within the European Project KoBaS (Knowledge Based Customized Services for Traditional Manufacturing Sectors Provided by a Network of High Tech SMEs) (KoBaS Consortium, 2006).

The project consortium involves 21 partners from 10 countries, in which 6 partners are European Institutions of R\&D: RPK (Germany), INESC Porto (Portugal), ITIA-CNR (Italy), EPFL and ETH Zurich (Switzerland), and Tekniker (Spain).

The project will make use of techniques such as Virtual Reality, 3D \& Discrete Events Simulation, Knowledge Based System and Finite Element Analysis, evolved, combined and optimized in their interaction thanks to a new brand of innovative technologies that are developed in the KoBaS project.

\subsection{The project vision}

The objective of KoBaS project is to create a network of high-tech small and medium enterprises, offering knowledge based services developed within the project to manufacturing companies operating in traditional sectors. Knowledge based services will be a customised integrated solution of KoBaS "components", which will be modules developed for specific purposes. The project vision is to provide a breakthrough in the current practices in the use of manufacturing machines, through the development of a set of generic tools of new conception. This will enable the quick customization of software solutions providing thus new advanced and powerful functionality, machine embedded, and supporting task and process 
planning, machine maintenance, training, and management. Thanks to this innovative platform, it will be possible for machine-tools and other production equipment to become intelligent, capable to communicate their environment and characteristics, to understand and work with digital models of the parts to produce and to enable an efficient dialog with their operators (KoBaS Consortium, 2004).

These generic tools will be published in a network of High-Tech SMEs. The manufacturing machine builder, confronted with the difficulty of the task and process planning of his equipment and willing to provide a new brand of powerful services to his customer, asks to the Network of High Tech SMEs for a customized software solution for his machine. The KoBaS Network thus provides, thanks to the new instruments and approaches developed within the project, a customized solution that will be embedded in the machine by the Machine Builder allowing him to sell an "intelligent machine" to the end user (KoBaS Consortium, 2004).

In this context, a Component (development tool) is a collection of methodologies, software tools and libraries that will be used by the Network of HTSMEs to generate a KoBaS Customized Solution. A Kobas Customized Solution is an integrated set of Black-Boxes (software components).

\subsection{The KoBaS components}

The basic components reflect the main aspects covered by the KoBaS approach in the use of a manufacturing machine, namely:

1. Graphical User Interface Construction Component: with this component the Network will be able to build and promptly configure a customized Man-Machine interface, based on low cost Virtual Reality, in order to make easily and naturally accessible all the functionalities and services to the end-user.

2. Rule-Based Knowledge Core Construction Component: this component is meant to provide the Network with the possibility to customize, for every manufacturing sector/machine confronted, a Rule-based Knowledge Base (process and geometry related), in order to support the machine task programming, configuration, maintenance and training through a knowledge based system.

3. Experience Data Base Construction Component: this component is used by the High Tech SMEs to build a Knowledge DB of past experience related with the Process handled. Once the cases are acquired and formalized, this module provides the methods for knowledge access and reuse. This module operates in strict connection with the Rule-Based Knowledge Core Construction Component.

4. Part Program Creator Component: with this module, the High Tech SMEs Network will be able to quickly provide a customized solution for the offline programming of the machine. For the part program creation, this module relies on the information provided by the knowledge base.

5. 3D Simulation Construction Component: This component is used to create a customized simulation environment. It is meant to move the machine into the virtual environment as it would do in the real world, providing the framework to display the machine tasks or the training and maintenance procedures.

6. Finite Element Analysis (FEM) Component: with this Component, the Network builds a customized FEM analysis. It provides pre and post processing analysis for the process performed by the manufacturing machine under consideration, giving a feedback on the performance of the Production Process. 
7. Maintenance and Diagnosis Component: with this component, the Network will be able to build a customized maintenance service for the specific machine. The solution provided is meant to prevent failures, to suggest intelligent maintenance, to demonstrate maintenance procedures. This module should grow wiser (as the rule related modules) as it's used.

8. Training module Construction Component: the Network will use this component to develop a training module for the machine studied, intended to provide training support for the end-user of the machine. The component will also use the other components capabilities (such as simulation) to provide a training virtual environment for the machine end-user

9. Machine Configuration and Mechatronics Construction Component: this component will allow the SMEs Network to develop solutions for the configuration of the machine studied, according to final user needs.

10. Build-in Management Functions Construction Component: this component is meant to provide the network with the ability to develop customized solutions for the management function of the machine. (cost planning, scheduling and optimization)

11. Integration Component: this component is used to build the framework for the solutions proposed, ensuring that all the other modules can communicate, and establishing standards for the methods and classes for every customized solution realized. Within this Component also the data structure and data model for the specific solution will be defined.

Every component is intended to provide the proper instruments and methodology in order to enable an easy customization of machinery according the specific requirements to fulfil in the scope of $\mathrm{KoBaS}$. As an example, the component related to maintenance functionalities, should allow the customization of a specific maintenance solution based on machine monitorization (by sensors), relevant signal analysis, automatic failure detection and diagnosis, alarms and ordinary and extraordinary maintenance plan. The next section describes in particular the Maintenance Customization Solution.

\section{THE KOBAS MAINTENANCE SOLUTION}

High maintenance costs and production losses due to machine breakdowns are pressing problems for today's manufacturers. In order to increase the reliability and availability of machines it is urgent to shift from scheduled maintenance to the new technology of constantly observing machine condition and predicting its working condition in advance. This is one of the main objectives of the KoBaS Maintenance Solution.

The great majority of the machines produced by European manufacturers do not have built-in monitoring, diagnosis, simulation and analysis abilities that would help the user to identify the proper course of actions nor do they have an intelligent machine capable of assuring its service.

Furthermore, new technologies in sensors recently under development will bring an added dimension to the machine "intelligence" that will need to be dealt with. Also, the openness, the suitability to the manufacturing equipments and the integration with other $\mathrm{KoBaS}$ components, will allow an easy adaptation to new machines and will provide a more natural and intuitive interaction with the operator 
namely, 3D simulation to machine tests and diagnosis, maintenance tasks supported by training, and dynamic and adaptable behaviour through experiences learned from past situations.

This openness will facilitate the management of shorter lifecycles for machine producers and will make these advanced maintenance concepts reachable to smaller machine producers.

\subsection{Creation of a Maintenance Customized Solution}

As depicted on Figure 1, the Maintenance Construction Component is used by the HT-SMEs to create the Maintenance Black-Box (BB). The main propose of this BB is to implement a customized maintenance management solution supporting the machine's maintenance operations (see above sub-section).

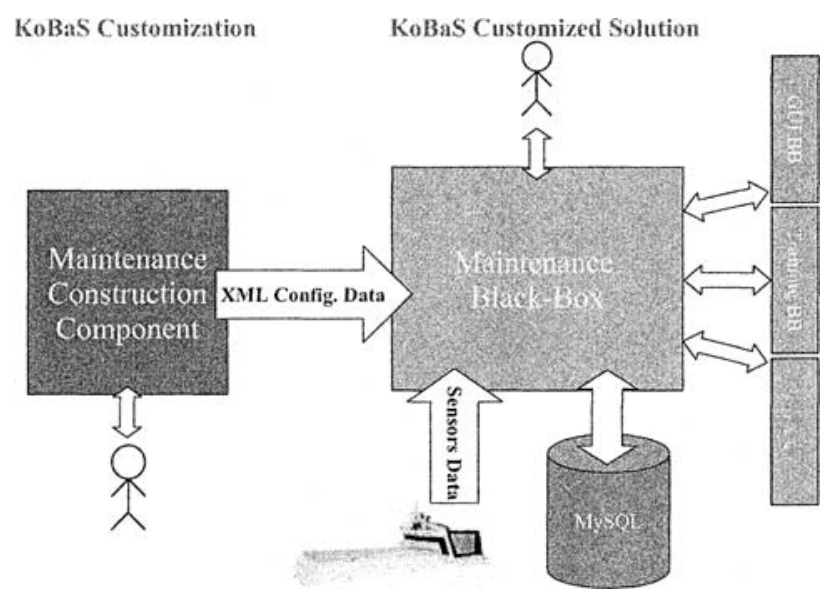

Figure 1: Creation of a Maintenance Customized Solution.

The Maintenance Black-Box is created through a set of XML configuration files and it is integrated with other $\mathrm{KoBaS} \mathrm{BB}$ as a solution. This solution will be embedded in the machine. The customization phase of the Maintenance BB involves the specification of the several structured information, such as: characterization of the machine, its modules, components and hierarchy; characterization of the machine/components failures; characterization of the preventive maintenance works, including the identification of the maintenance tools to do the work, the spare parts and components to replace and the maintenance training procedures to help the maintenance preventive intervention; characterization of machine parameters; characterization of machine processes and relation with the monitorization parameters. 


\subsection{The Maintenance BB}

Figure 2 presents the architecture overview of the Maintenance BB. This architecture comprises several modules reflecting the set of functionalities considered in the component.

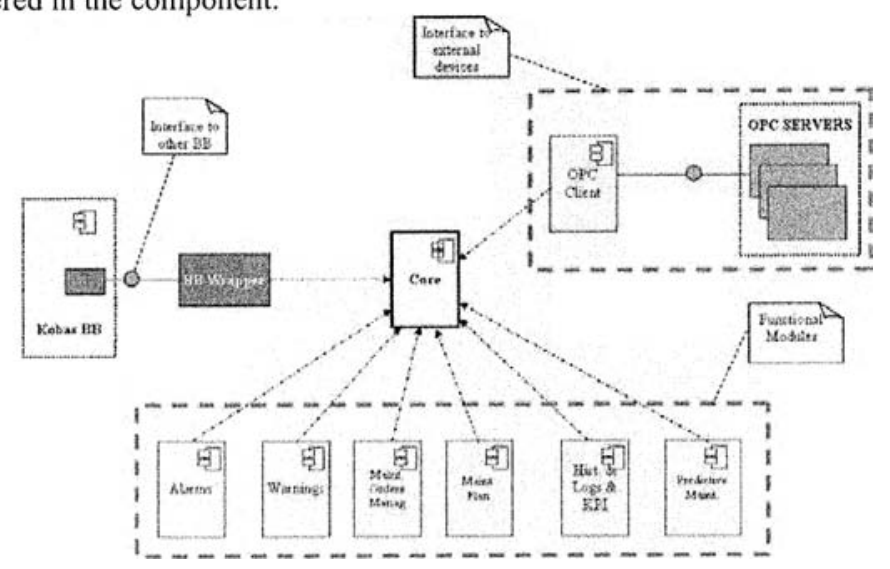

Figure 2: Architecture overview of Maintenance Component.

\section{Interaction with the machine controllers}

The Maintenance BB must have a tight interaction with sensors and transducers embedded on the machine in order to receive real-time data and thus provide functionalities related to detection and prediction of machine failures. The approach followed was to design a solution that will act as a client accessing the equipment main controller through a standard interface.

The use of a standard interface enables the interoperability between automation/control applications, field systems/devices and business/office applications. It provides a standard method for exchanging information between the machine controllers and the KoBaS Maintenance $\mathrm{BB}$ across a wide range of platforms and involving different technologies. One of the main requirements is the assurance that software is written independently of the type of controller considered.

In factory automation domain, the OPC protocol has been proposed as a standard method for exchanging data between automation elements. This nonproprietary technical specification defines a set of standard interfaces originally based upon Microsoft's OLE/COM/DCOM technology specification (OPC Foundation Website). It is nowadays the preferred connectivity medium into enterprises, and supported by major providers of control systems and industrial instrumentation (Hong and Jianhua, 2006). Currently, OPC XML-DA specification which builds on the OPC Data Access specifications to communicate data in XML, is available. It bases on the web services standards XML, SOAP and WSDL and standardizes the SOAP messages exchanged between clients and server (Zampognaro, 2004). The standardization of messages instead of an application interface allows the implementation on different operating systems and does not base exclusively on Microsoft's COM/DCOM. In this context, the OPC XML-DA standard specification was selected. 
Concerning the physical infrastructure, the approach should support two different scenarios:

- OPC Client(s) and Server(s) in the same node - OPC clients and servers run as concurrent processes in the same computer (node);

- OPC Client and Server in different nodes - in this situation OPC clients and servers run in different computer platforms.

The same equipment (machine) can involve more than one OPC server running in the same node or in different nodes (physically located in the machine). Moreover, it is possible to have a direct connection between OPC Server and devices as well as an indirect connection through one or several fieldbus networks.

\section{The Predictive Maintenance module}

The Predictive Maintenance Module, which is condition monitoring based, will support monitoring, trigger conditions, and short and long term forecasting for slowly changing sensors. To perform the monitorization the $\mathrm{OPC}$ client will make data requests to the Industrial Machine for a specific sensor at specified time intervals. The read value is then appended to a history buffer and applied to the Trigger Condition and Forecast sub-modules.

In the Trigger Condition sub-module the read value is compared with predefined threshold that (if exceeded) can trigger warnings or failures. In this submodule the values from multiple sensors are also conjunctly evaluated using a Knowledge Base Rules Engine, looking for conditions that can indicate a possible machine or sensor failure, or help diagnose an existing problem. The recent history of the sensor values can also be graphically displayed against the target and threshold limits.

The recent history (minutes) of the sensor values form the basis of a non-linear adaptive ANN-based prediction sub-module, whose function is to predict the sensor value in the very near future and compare the predicted values with predefined thresholds. This module makes it possible to avoid failures originated from punctual and otherwise unexpected situation, such as a suddenly clogged coolant or pressure line.

A similar technique will monitor the long-term trends of a machine component or sub-system monitored by a given sensor. Past sensor values in the range of weeks to months will reveal any long-term trend and, through interaction with the Maintenance scheduler, anticipate or delay a planned Maintenance operation, eliminating unnecessary downtimes and unnecessary parts replacement. Seasonal trends must be detected if possible and not deploy Maintenance operations.

The earlier warnings will be integrated into the Trigger Condition sub-module where, together with other sensor values, it will help to diagnose the problem in advance.

The above described techniques are used for slowly changing data, such as temperature, pressure, etc, but for rapidly changing data sensors, such as accelerometers, instantaneous motor current, etc., further pre-processing steps are needed. This kind of sensors is more effective in the frequency domain, forming the basis of Vibration Analysis Techniques, and for this reason its values will be acquired and process in accordance. Depending on the sensor and techniques to use, the acquired signal will undergo a Fast Fourier or Wavelet Transforms and a Feature 
Selection/Extraction step before being applied to the Trigger or Forecast submodule.

As with slowly changing sensors, rapidly changing sensors will be acquired at specified time intervals, but a burst of hundreds of equally and rapidly sampled values will be read instead.

\section{The Warnings and Alarms modules}

The Maintenance BB generates a warning when the monitorization detects one or more parameter value over the warning limits previously configured. The Predictive Maintenance (short or long-term forecast) also generates a warning when it predicts that one or more parameters will come over the warnings limits previously configured. If both monitorization and forecast detect a warning in the same prediction interval only one warning is generated.

The Maintenance BB generates an alarm each time a machine failure occurs (a Corrective MWO or a Predictive MWO is created) or near the planed date of the Systematic Maintenance intervention. Upon the occurrence of an alarm or warning the system waits by the operator acknowledge. The event remains active during the configured time-out. The event will be repeated a number of times according to the configured frequency if there is no the operator acknowledge. When the operator acknowledges the event the acknowledge date is recorded on the system. The system will provide different views over the alarms and warnings data.

\section{The Maintenance Orders Management module}

The system allows the management of Corrective MWO, Systematic MWO and Predictive MWO. The machine typical failures should be configured first by the Machine Builder during the KoBaS customization phase and completed later by the End User, according to his experience, during the machine runtime. The same configuration procedure should be made for the maintenance jobs. The Corrective MWO is created by the system when a failure occurs in the machine. The Systematic MWO could be created by the Knowledge Engineer in the KoBaS customization phase or by the End User during the machine operation. The Predictive MWO is created by the system when it predicts the failure occurrence.

When the MWO is created it includes all the information that will be necessary to repair the machine. After the maintenance intervention (according to the End User records and updates) it also includes all the information related with the intervention, namely the maintenance time, the repair time and the components replaced.

\section{The Maintenance Plan module}

The Systematic MWO are planned (ordinary maintenance plan) according to the knowledge of the Machine Builder, in the KoBaS customization phase, and in the runtime, according to the experience of the End User. These MWO are periodic and generated automatically by the system according to the maintenance period. The Predictive MWO are planned (extraordinary maintenance plan) by the system according to the failure estimation. The ordinary and the extraordinary maintenance plan are validated by the machine scheduler. 


\subsection{Interaction with other KoBaS Black-Boxes}

The Maintenance BB interacts with other Black-Boxes (BB) using its services (represented by interfaces) in order to obtain essential data necessary to process information to be presented by the graphical user interface. The GUI BB allows the creation of the graphical user interface.

During the maintenance intervention the End User could ask the system about the best procedures to make the intervention. The Training BB is the front end for the end user offering all functionalities to search for maintenance requested training and managing the execution. Before or during the maintenance intervention the End User could access to the Simulation function to test and to underline situations related with the failure, like "put this object in this position", "change the colour of this body" and others. The Simulation BB offers a virtual environment representing the manufacturing machine helping the end user on the failure diagnosis.

The Rule Based BB provides rule based knowledge for the other black-boxes namely the Maintenance $\mathrm{BB}$, in order to allow the detection and diagnosis of machine abnormalities and failures.

The Maintenance BB extracts cases related with the maintenance history in order to help in the failure diagnoses. The Experience Data Base BB is an easy-touse tool for the creation of a customized case base reasoning expert system.

The Built-in management BB generates production schedules. The Maintenance $\mathrm{BB}$ interacts with it to inform and to validate the dates of planned maintenance.

\section{DEVELOPMENT AND IMPLEMENTATION}

By definition, a KoBaS maintenance solution is an integrated set of black-boxes (software components) targeted to implement maintenance related functionality. Two approaches have been considered to deploy such a KoBaS solution in an equipment machinery: installation of the software in the computer embedded in the equipment machinery itself (and responsible for controlling the machine); installation of the software in a separate computer with a communication channel with the machine's control computer.

Despite the approach taken by the Machine Builder, the software must run in several computational platforms, being the Linux and Windows operating system the minimum requirement. In this context, the Java programming language and the Java 2 Standard Edition constituted the basic ingredients to build a KoBaS solution. A KoBaS solution is then an integrated set of Black Boxes doing their work within a Java virtual machine.

On the other hand, Open Source software constituted another major choice. The development of a KoBaS solution should make use of Open Source software whenever possible. In this context, several tools and frameworks were selected to ease the software development. The MySQL database management system (MySQL, 2005) is being used in the Maintenance solution in order to assure the required data persistency functionality. Access to the data base is implemented by the JDBC API and, on top of it, by the Hybernate framework (Hibernate, 2005). This framework maps Java objects in the object-oriented world to data records in the 
relational data base world facilitating thus the development of the Maintenance persistency function. The major development tool is the Eclipse IDE (Eclipse Website).

\section{CONCLUSIONS}

The KoBaS Project (Knowledge Based Customized Services for Traditional Manufacturing Sectors Provided by a Network of High Tech SMEs) is an European project under the Sixth Framework Programme that tends towards a substantial improvement in equipment and industrial machinery.

The customization of specific solutions required by the equipment manufacturers according to the requisites demanded by the market will be supplied by a network business model, made of technology-based SMEs. Each KoBaS customized solution is an integrated set of Black-Boxes generated from software components by the Network of HT-SMEs.

The Maintenance Solution is one of the KoBaS solutions that allow an easy adaptation to new machines and provides a more and intuitive interaction with the operator. It was conceived to provide, machine monitorization, failures detection, failures prediction, alarms, maintenance simulation, training, ordinary and extraordinary maintenance plan and Work Orders management. To fulfil these functions the solution includes the Maintenance $\mathrm{BB}$, based on prediction maintenance condition, and other $\mathrm{KoBaS} \mathrm{BB}$, on one hand, to help in the failures diagnostic, and on the other hand, to supply to the end user maintenance training, simulation, and maintenance plan.

The innovative concept presented on this paper allows a greater integration between the machine performances and the related process parameters and promotes the evolution of SMEs operating in traditional industrial sectors, reinforcing and integrating competitiveness, innovation and sustainability.

\section{ACKNOWLEDGEMENT}

This work has been partly funded by the European Commission through Project KoBaS (No. 505339-2 FP6-2002-NMP-2). The authors wish to acknowledge the Commission for their support and $\mathrm{KoBaS}$ project partners for their contribution during the development of various ideas and concepts presented in this paper.

\section{REFERENCES}

1. Eclipse Website, htp: Www eclipse.org

2. Hibernate. Hibernate Open Source Project, http //www hibernute.org/, accessed 10.1.2006.

3. KoBaS Consortium (2004). KoBaS Description of Work. Technical Report. KoBaS European project 505339-1.

4. KoBaS Consortium (2006). KoBaS Web site, http://www.kobasproject.com, accessed 10.1.2006.

5. MySQL. MySQL Project, http://java.sun.com/jdbc, accessed 10.1.2006.

6. The OPC Foundation Website, www.opcfoundation org

7. Hong $X$, Jianhua $W$. Using standard components in automation industry: $A$ study on OPC Specification. Computer Standards \& Interfaces 28 (2006) 386-395

8. Zampognaro, Francesco. Specifiche ed Implementazione di un Middleware per Applicazioni RealTime Mediante la Tecnologia Web Service. Master Thesis - Universita degli studi di Roma, 2004 